\title{
NOUVELLE
}

\section{microARN et inactivation de la fonction gonadotrope hypophysaire}

Jérôme Lannes, David L'Hôte, Ambra Fernandez-Vega, Ghislaine Garrel, Jean-Noël Laverrière, Joëlle Cohen-Tannoudji, Bruno Quérat
Sorbonne Paris Cité, Université Paris-Diderot, CNRS, Inserm, Biologie fonctionnelle et adaptative UMR 8251, Physiologie de l'axe gonadotrope, U1133, 5 , rue Thomas Mann, 75205 Paris Cedex, France. bruno.querat@univ-paris-diderot.fr
> La neuro-hormone hypothalamique gonadotropin-releasing hormone ( $\mathrm{GnRH}$ ) joue un rôle crucial dans la fonction de reproduction en stimulant la synthèse et la sécrétion des gonadotropines luteinizing hormone ( $\mathrm{LH})$ et follicle-stimulating hormone (FSH) par l'hypophyse. À leur tour, LH et FSH régulent l'activité des gonades en stimulant la gamétogenèse et la sécrétion d'hormones stérö̈des sexuelles [1]. Cependant, une stimulation prolongée par la $\mathrm{GnRH}$ conduit à la désensibilisation des cellules gonadotropes hypophysaires et à l'arrêt de la production des gonadotropines [2]. Ce phénomène est bien connu des cliniciens qui cherchent à réduire, de façon réversible, la production des stéroïdes sexuels dans certaines pathologies comme les cancers dépendants de ces stéroïdes ou le syndrome des ovaires polykystiques $^{1}$ [3]. Les mécanismes responsables de cette désensibilisation sont encore mal compris. Dans l'étude que nous rapportons ici [4], nous avons cherché le rôle que pourraient jouer les micro-ARN (miARN) dans ces mécanismes. Les miRNA régulent en effet le fonctionnement de nombreux gènes en induisant la dégradation ou la séquestration de leurs ARN messagers dans les complexes RISC (RNA-induced silencing complex). Leur déré- $\quad(\rightarrow)$ Voir la Nouvelle gulation est à l'origine de J. Cavaillé, $\mathrm{m} / \mathrm{s}$ $n^{\circ} 4$, avril 2004, de très nombreuses page 399, et la Synpathologies [5-7] thèse de S. Baulande $(\rightarrow)$.

et al., $m / s n^{\circ} 3$, mars 2014, page 289

\footnotetext{
${ }^{1}$ Trouble hormonal touchant jusqu'à $10 \%$ des femmes dans lequel une production anormale d'androgènes perturbe la production d'ovules et provoque l'apparition de kystes ovariens.
}

Dans une première étude [8], nous avions montré que le miARN-132 (miR132) était fortement induit dans les cellules gonadotropes en réponse à la $\mathrm{GnRH}$ et que son augmentation était indispensable à l'induction de la production de FSH par la GnRH. À l'inverse, le miR-125b est, lui, fortement inhibé en réponse à la GnRH [9].

\section{miR-125b bloque la production des} gonadotropines induites par la GnRH Nous montrons dans cette étude que la surexpression de miR-125b empêche l'activation de la production des gonadotropines par la GnRH, aussi bien dans des cultures primaires de cellules hypophysaires de rat que dans la lignée murine de cellules pituitaires gonadotrope L $\beta$ T2 [4]. À l'inverse, l'inhibition de miR-125b entraîne une augmentation de la production des gonadotropines, montrant que le taux de ce miARN présent à l'équilibre (dans la cellule non stimulée) est suffisant pour inhiber la production de ces hormones dans la cellule gonadotrope. Le miR-125b bloque l'une des deux principales voies de signalisation par lesquelles la GnRH stimule la production des gonadotropines [10], celle résultant du couplage du récepteur de la $\mathrm{GnRH}$ à la protéine $\mathrm{G} \alpha q / 11^{2}$. La surexpression de miR-125b conduit en effet à la séquestration des transcrits de plusieurs effecteurs de cette voie de signalisation dans les complexes RISC (mesuré par $\mathrm{PCR}$ [polymerase chain reaction] quantitative après immunoprécipitation des

\footnotetext{
${ }^{2}$ Sous-unité d'une protéine $\mathrm{G}$ activant la phospholipase C.
}

complexes) et à leur dégradation (évaluée par mesure de la quantité cellulaire de ces transcrits). Le taux d'expression protéique de ces effecteurs, déterminé par western blot, est également diminué de façon significative. À l'inverse, l'inhibition de miR-125b entraîne une augmentation de l'expression de ces protéines. Sachant que de nombreux autres récepteurs sont couplés à $\mathrm{G} \alpha q / 11$, miR125b pourrait donc participer à la régulation de la signalisation que ces récepteurs induisent dans de nombreux autres types cellulaires.

Il est intéressant de noter que le miR125 b n'affecte pas, en revanche, la signalisation de la GnRH s'exerçant via le couplage à la protéine $\mathrm{G}_{\alpha s^{3}}$.

\section{miR-125b et miR-132 sont liés par une} boucle de régulation

Alors que la quantité à l'équilibre de miR-125b est suffisante pour bloquer la signalisation induite par la voie $\mathrm{G} \alpha q / 11$ (Figure 1, voie 1), la stimulation de la voie $G \alpha s$ par la $\mathrm{GnRH}$ est maintenue et permet l'activation de la protéine kinase A (PKA ; Figure 1, voie 2). Parmi les cibles de la PKA, nous avons identifié une ARN-méthyltransférase, NSun2 (NOP2/Sun family member 2) dont la phosphorylation par la PKA active le transfert d'un groupement méthyle sur miR-125b (Me-miR-125b) (Figure 1, voie $3)$. Cette méthylation entraîne la diminution de la quantité de miR-125b dans

\footnotetext{
${ }^{3}$ Sous-unité d'une protéine $G$ activant la voie dépendante de I'AMPc (adénosine monophosphate cyclique), ou voie de l'adénylate cyclase, impliquée dans la communication cellulaire.
} 


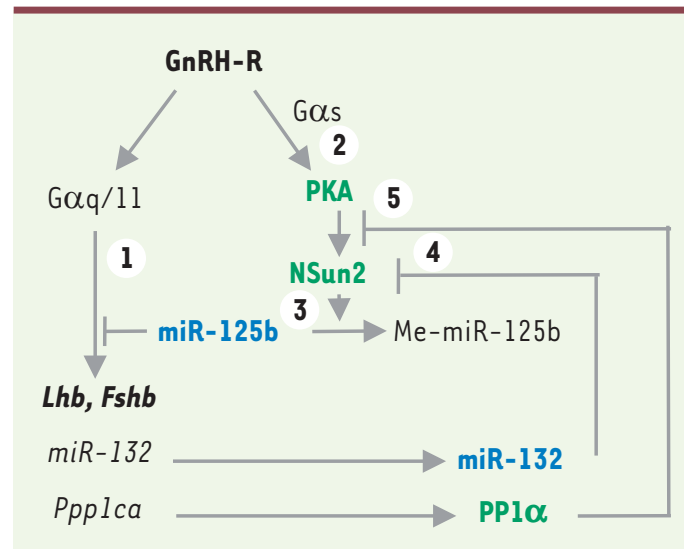

Figure 1. Représentation schématique de la boucle de régulation entre miR-125b et miR-132 dans la cellule gonadotrope. (1) miR-125b bloque la signalisation activée par le couplage du récepteur de la $\mathrm{GnRH}$ (gonadotropin releasing hormone) à la protéine $\mathrm{G} \alpha q / 11$. (2) Le couplage du récepteur de la GnRH à la protéine Gas active la PKA (protéine kinase A) qui active à son tour l'ARN méthyl-transférase Nsun2 (NOP2/sun family member 2) par phosphorylation. (3) L'activation de Nsun2 provoque la méthylation de miR-125b (Me-miR-125b) et sa diminution, permettant la signalisation $\mathrm{G} \alpha q / 11$. Ceci se traduit par l'activation transcriptionnelle des gènes Lhb (luteinizing hormone beta subunit), Fshb (follicle stimulating hormone beta subunit), miR-132 et de la sous-unité catalytique de la PPl $\alpha$ (protein phosphatase type l alpha), PpplCa. (4) En retour, miR132 inhibe la traduction de Nsun2 et (5) PPl $\alpha$ inactive Nsun2 par déphosphorylation, ce qui conduit à une nouvelle augmentation de la quantité cellulaire de miR-125b et à un nouveau blocage de la voie $\mathrm{G} \alpha q / 11$.

A miR-125b (RISC)

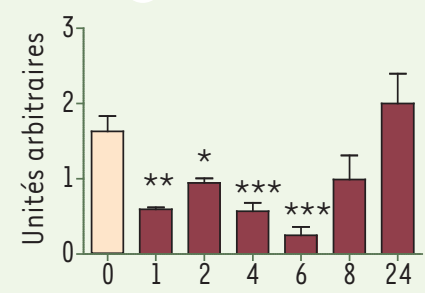

B miR-132 (RISC)

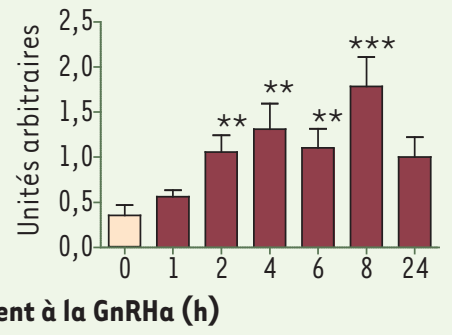

C Protéine NSun2

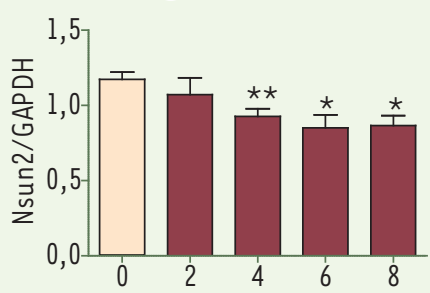

D p-NSun2/NSun2

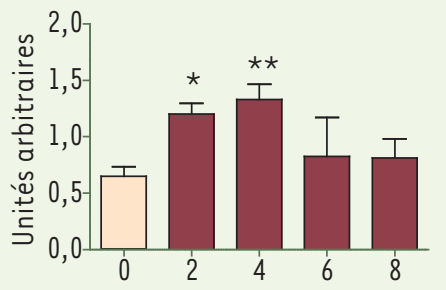

Durée du traitement à la GnRHa (h)

F Lhb

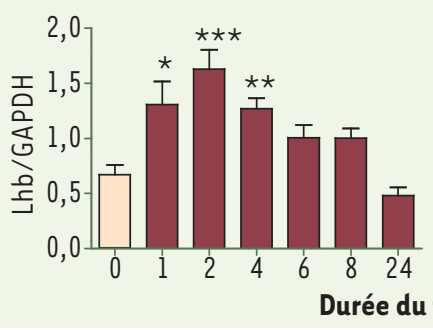

G Fshb

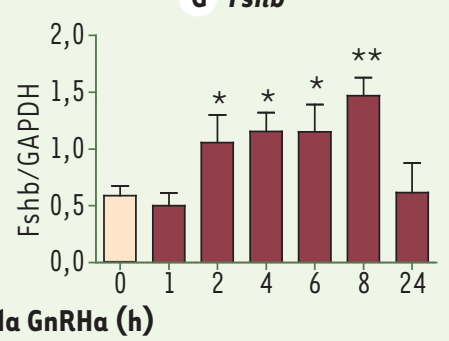

$\varepsilon \operatorname{PpplCa}(\mathrm{PP} 1)$

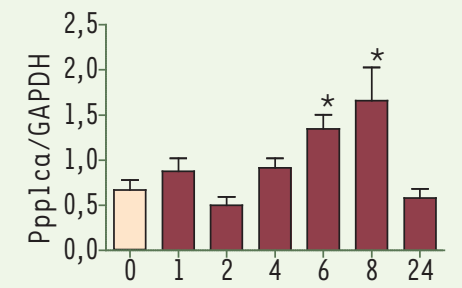

Figure 2. Analyse cinétique d'un traitement par la GnRH. Les cellules gonadotropes murines $L \beta T 2$ sont soumises à un traitement par l'agoniste de la GnRH (GnRHa), la triptoréline (Decapepty|TM), à $10 \mathrm{nM}$ pendant $24 \mathrm{~h}$. Le niveau de miR-125b (A) et de miR-132 (B) dans les complexes RISC (RNA-induced silencing complex), permettant d'évaluer la fraction active des miARN, est quantifié par PCR (polymerase chain reaction) quantitative après immunoprécipitation des complexes et est rapportée à la quantité totale de ces mêmes miARN dans la cellule. La quantité de protéines Nsun2 (NOP2/sun family member 2) est mesurée par analyse en Western blot à l'aide d'un antisérum spécifique et rapportée à la quantité de la protéine GAPDH (glyceraldehyde-3 phosphate deshydrogenase) (C). La fraction phosphorylée de la protéine Nsun2 est mesurée après fractionnement sur colonne d'enrichissement en phosphoprotéines et rapportée à la quantité totale de Nsun2 (D). Les niveaux des messagers de la sous-unité catalytique de PPl $\alpha$ (protein phosphatase type 1 alpha), PpplCa (E), de Lhb (luteinizing hormone beta subunit) (F) et Fshb (follicle stimulating hormone beta subunit) (G) sont mesurés par PCR quantitative et rapportés au niveau de l'ARNm codant la GAPDH. Les valeurs moyennes sont représentées \pm SEM (standard error of the mean). ${ }^{*}: P<0,05 ; * \star: P<0,01 ; * \star *: P<0,001$. 
la cellule et sa sortie des complexes RISC, ce qui permet la levée de la répression de la voie $\mathrm{G} \alpha q / 11$. Les effecteurs de la signalisation Gaq/ll peuvent alors agir au niveau transcriptionnel pour stimuler la production des gonadotropines. De façon remarquable, ces effecteurs sont également responsables de l'augmentation de miR-132 qui diminue la production de NSun2 (Figure 1, voie 4) en favorisant la séquestration de son transcrit dans le complexe RISC, et de la sous-unité catalytique de la protéine phosphatase PPl $\alpha, \operatorname{PpplCa}$, qui déphosphoryle NSun2, substrat de la PKA (Figure 1, voie 5). Ces deux effets lèvent ainsi l'action inhibitrice de Nsun2 sur miR-125b, permettant l'augmentation de son taux dans la cellule. Cette augmentation de miR-125b, qui favorise le blocage de la voie $\mathrm{G} \alpha q / 11$, est à l'origine de la diminution de la quantité de miR-132. L'ensemble de ces résultats révèle ainsi l'existence d'une boucle de régulation établie entre miR-125b et miR-132 qui tend à les maintenir à leur niveau d'équilibre.

La stimulation prolongée par la GnRH active la boucle de régulation qui provoque l'arrêt de son action Une analyse cinétique de l'ensemble de ces processus nous a permis de décrypter le mécanisme de désensibilisation de la cellule gonadotrope en réponse à une stimulation prolongée par la $\mathrm{GnRH}$ (Figure 2). Lors de la phase d'induction par la GnRH (4 à 6 h de traitement), l'activation de la voie Gos inactive miR125b, dont le taux atteint son niveau minimal dès $1 \mathrm{~h}$ et reste bas jusqu'à $6 \mathrm{~h}$
(Figure 2A). Cette diminution rapide de miR-125b lève l'inhibition qu'il exerce sur la voie $\mathrm{G} \alpha q / 11$ et, en conséquence, permet l'induction des gènes des gonadotropines Lhb (luteinizing hormone beta subunit) (Figure 2F) et Fshb (follicle stimulating hormone beta subunit) (Figure 2G). Cela se traduit également par une augmentation des quantités de miR-132 (Figure 2B) et de la sous-unité catalytique PpplCa (Figure 2E) qui, à leur tour, sont à l'origine de la diminution (Figure 2C) et de l'inactivation (Figure 2D) de NSun2 par déphosphorylation, après 4 à $6 \mathrm{~h}$ de traitement, et le retour de miR-125b à son état d'équilibre (entre 8 et 24 h). Ce mécanisme réprime à nouveau la voie $\mathrm{G} \alpha q / 11$ et, en conséquence, la production des gonadotropines. Le niveau d'expression de Fshb reste fort tant que le niveau de miR-132 est élevé, comme cela avait été montré dans nos travaux précédents [8]. L'augmentation de l'expression de Lhb est plus temporaire. Les gonadotropines $\mathrm{LH}$ et FSH retrouvent leur niveau minimal après $24 \mathrm{~h}$ de stimulation, un temps compatible avec ce qui est observé in vivo lors de traitements prolongés par la $\mathrm{GnRH}$ chez le rat [11].

Notre étude montre ainsi pour la première fois le rôle crucial joué par une boucle de régulation entre deux miARN ${ }^{4}$. Cette boucle miR-125b/miR132 est directement impliquée dans le mécanisme de la désensibilisation de la réponse à la $\mathrm{GnRH}$. Le fait que les acteurs de cette boucle de régulation

\footnotetext{
${ }^{4}$ Les détails de cette étude sont disponibles dans l'article original [4].
}

(miR-125b et miR-132, PKA, Nsun2 et $P P l \alpha$ ) soient retrouvés dans de très nombreux types cellulaires laisse penser qu'elle pourrait jouer un rôle plus ubiquitaire. $\diamond$

microRNA and inactivation of the

pituitary gonadotrope function

\section{LIENS D'INTÉRÊT}

Les auteurs déclarent n'avoir aucun lien d'intérêt concernant les données publiées dans cet article.

\section{RÉFÉRENCES}

1. Seeburg PH, Mason AJ, Stewart TA, Nikolics, K. The mammalian $\mathrm{GnRH}$ gene and its pivotal role in reproduction. Rec Progr Horm Res 1987 ; 43 : 69-98.

2. Belchetz PE, Plant TM, Nakai $Y$, et al. Hypophysial responses to continuous and intermittent delivery of hypopthalamic gonadotropin-releasing hormone. Science $1978 ; 202: 631-3$.

3. Kiesel LA, Rody A, Greg RR, Szilagyi A. Clinical use of GnRH analogues. Clin Endocrinol 2002 ; 56 : 677-87.

4. Lannes J, L'Hôte D, Fernandez-Vega A, et al. A regulatory loop between miR-132 and miR-125b involved in gonadotrope cells desensitization to GnRH. Sci Rep $2016 ; 6$ : 31563.

5. Cavaillé J. Des microARN comme s'il en pleuvait... Med Sci (Paris) $2004 ; 20: 399-401$.

6. Baulande $S$, Criqui A, Duthieuw M. Les microARN circulants, une nouvelle classe de biomarqueurs pour la médecine. Med Sci (Paris) 2014 ; 30 : 289-96.

7. He L, Hannon GJ. MicroRNAs: small RNAs with a big role in gene regulation. Nat Rev Gen 2004 ; 5 : 522-31.

8. Lannes J, L'Hôte D, Garrel G, et al. A microRNA-132/212 pathway mediates $\mathrm{GnRH}$ activation of FSH expression. Mol Endocrinol 2015 ; 29 : 364-72.

9. Godoy J, Nishimura M, Webster NJ. Gonadotropinreleasing hormone induces miR-132 and miR-212 to regulate cellular morphology and migration in immortalized LbetaT2 pituitary gonadotrope cells. Mol Endocrinol $2011 ; 25: 810-20$.

10. Liu F, Usui I, Evans LG, et al. Involvement of both $G(q / 11)$ and $G(s)$ proteins in gonadotropin-releasing hormone receptor-mediated signaling in LbetaT2 cells. J Biol Cell 2002 ; 277 : 32099-108.

11. Lerrant $Y$, Kottler ML, Bergametti F, et al. Expression of gonadotropin-releasing hormone $(\mathrm{GnRH})$ receptor gene is altered by $\mathrm{GnRH}$ agonist desensitization in a manner similar to that of gonadotropin betasubunit genes in normal and castrated rat pituitary. Endocrinology 1995 ; 136 : 2803-8.

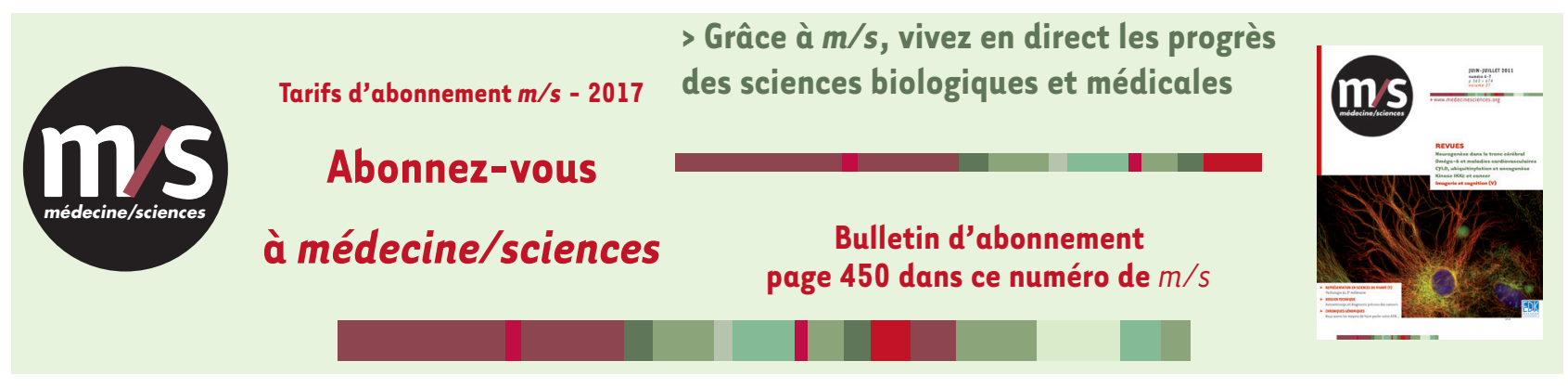

